\title{
Comparison of modified transumbilical laparoendoscopic single-site nephroureterectomy and retroperitoneal laparoscopic nephroureterectomy: initial experience
}

\author{
Yang Shen ${ }^{1}$, Hesong Ye ${ }^{2}$, Qingyi Zhu ${ }^{1}$, Jian Su${ }^{1}$, Chen Zhu ${ }^{1}$, Zhonglei Deng ${ }^{1}$, Long Ma ${ }^{1}$, Lin Yuan ${ }^{1}$ \\ ${ }^{1}$ Department of Urology, Affiliated Hospital of Nanjing University of Chinese Medicine, Jiangsu Provincial Hospital of Chinese Medicine, \\ Nanjing, Jiangsu, China \\ 2Department of Urology, The Second Affiliated Hospital of Nanjing University of Chinese Medicine, Jiangsu Provincial Second Chinese \\ Medicine Hospital, Nanjing, Jiangsu, China
}

Videosurgery Miniinv 2020; 15 (1): 199-207 DOI: https://doi.org/10.5114/wiitm.2019.87492

\begin{abstract}
Introduction: Owing to the development of the laparoendoscopic single-site (LESS) procedure, transumbilical LESS nephroureterectomy (LESS-NU) has become a new approach for treating upper tract urothelial carcinoma.

Aim: The aim of this study is to introduce a modified LESS-NU procedure with bladder cuff excision for treating upper tract urothelial carcinoma (UTUC). We compared its clinical efficacy and postoperative outcomes in terms of follow-up time with traditional retroperitoneal laparoscopic nephroureterectomy (RL-NU).

Material and methods: From May 2014 to May 2019, we performed nephroureterectomy on 42 patients using the retroperitoneal approach and a modified LESS approach. A retrospective analysis was conducted for the evaluation of the clinical and postoperative outcomes between the two groups.

Results: The study included 25 LESS-NU and 17 RL-NU patients. All the procedures were completed successfully. The LESS-NU group had a significantly shorter mean operative time than the RL-NU group (204.4 min, $236.18 \mathrm{~min}$, $p=0.005)$. The differences in skin incision length $(2.88 \mathrm{~cm}, 8.94 \mathrm{~cm}, p<0.001)$ and oral analgesic dose $(n=1.12$, $n=2.75, p<0.001)$ between LESS-NU and RL-NU were statistically significant.

Conclusions: Modified LESS-NU is a feasible and safe procedure. Compared with the retroperitoneal laparoscopic approach, the single-site approach did not alter the patients' position. LESS-NU is a better procedure for treating UTUC than RL-NU in terms of cosmetic result and postoperative pain.
\end{abstract}

Key words: laparoendoscopic single-site, minimally invasive surgery, nephroureterectomy, upper tract urothelial carcinoma.

\section{Introduction}

Upper urinary tract urothelial carcinoma (approximately $5-10 \%$ of all urothelial carcinoma cases) rapidly develops in the renal pelvis and ureter [1]. Radical nephroureterectomy serves as the main treatment for the disease, and entire excision including the bladder cuff remains the gold standard [2]. In the 1990s, the laparoscopic technique began to be used in urology surgery and combined with nephroureterectomy [3]. With technological innovations, the retroperitoneal approach has been widely used in nephroureterectomy because of its clinical efficacy. However, surgeons are not satisfied with the retroperitoneal approach and are eager to find safer

\section{Address for correspondence}

Prof. Lin Yuan, Department of Urology, Affiliated Hospital of Nanjing University of Chinese Medicine, Jiangsu Provincial Hospital of

Chinese Medicine, Nanjing, Jiangsu, China, e-mail: yuanlin47@163.com 
and more effective alternative procedures. Laparoendoscopic single-site (LESS) surgery, as a novel procedure, appeared by chance [4]. It is a critical stage in the evolution of laparoscopic surgery. In comparison with traditional retroperitoneal laparoscopic surgery, which requires $3-5$ incisions, each at least $1-2 \mathrm{~cm}$ in length, LESS decreases the potential morbidity risks of bleeding and wounds [5, 6]. Apart from the above-mentioned advantages, patients may suffer less postoperative pain [7, 8].

Although early papers have demonstrated the safety and feasibility of the LESS procedure, analysis and comparison between LESS nephroureterectomy (LESS-NU) and retroperitoneal laparoscopic nephroureterectomy (RL-NU) are lacking. Based on our initial experience of the LESS procedure [9-11], we designed our own modified LESS-NU procedure with bladder cuff excision. It not only reduced the difficulty of the procedure and the operating time but also prevented patients' intraoperative repositioning.

\section{Aim}

We aimed to introduce a modified LESS-NU procedure with bladder cuff excision designed by our department and compare the operative results between RL-NU and modified LESS-NU.

\section{Material and methods}

\section{Patients' selection}

From May 2014 to May 2019, 25 patients underwent modified LESS-NU and 17 patients underwent RL-NU. The patients we enrolled in the study were diagnosed with upper urinary tract urothelial cancer (UTUC). Diagnosis of these patients was accomplished by urinary tract ultrasonography, intravenous pyelography (IVP), computed tomography (CT), MRU, cystoscopy, and ureteroscopy in our hospital. All patients with non-transitional cell carcinoma (non-TCC), bladder tumor, clinical > T3 disease, and metastatic disease on the preoperative staging were excluded. None of these patients had adjuvant chemotherapy before surgery.

\section{RL-NU procedure}

The RL-NU procedure was carried out as previously reported $[12,13]$. After the induction of general anesthesia, the patients were placed in a lateral $90^{\circ}$ position on the operating table. A $1.5 \mathrm{~cm}$ inci- sion was made in the junction of the psoas edge and costal margin. A balloon was introduced and inflated to separate the retroperitoneal space. Then, 3-4 trocars were placed. After separating the kidney from the middle ureter to renal vein and artery, the patient was repositioned from lateral to supine. A new disinfecting and toweling process was performed. Subsequently, a large lower abdominal oblique incision was made to dissect the bladder cuff and lower ureter, and the intact specimen was extracted with a specimen bag. Finally, the surgery was completed after wound closure.

\section{LESS-NU procedure}

Under general anesthesia and endotracheal intubation, the patients were placed in a modified position, and the flank was elevated by $30-45^{\circ}$ and fixed on the operating table. The entire procedure was carried out in the same position in addition to minimal leaning and tilting of the operating table. First, access was obtained via an approximately $3 \mathrm{~cm}$ midline circumferential umbilical incision (Figure $1 \mathrm{~A})$. All the instruments were placed through the QuadPort (Innovex Bioscience, Shanghai, China) multichannel port (Figure 1 B). We insufflated carbon dioxide to maintain an intraperitoneal pressure of $15 \mathrm{~mm} \mathrm{Hg}$ and settle the pneumoperitoneum.

After identification of the anatomical sign, the paracolic sulci were opened. The entire colon slid downward due to gravity, thereby exposing the renal hilum. The renal fascia was incised, and the renal vein was identified. Behind the renal vein, the renal artery was exposed. Herein, the additional blood vessels were considered. EndoGIA was used to cut the renal pedicle after the renal artery was separated from the vein (Figure $2 \mathrm{~A}$ ).

The kidney was sharp-separated gradually. After lifting up the inferior pole of the kidney, the renal pelvis and ureter were divided away from the paracolic. The ureter was lifted, and the tension was maintained. Then, the intact ureter was divided downward gradually. In this process, the surgeons just have to adjust the operation direction instead of the patients' position (Figure $1 \mathrm{C}$ ). The bladder cuff was dissected around the ureteric orifice. Then, the bladder defect was closed with V-loc sutures by running stitches and tightening (Figure 2 B). The suture tail was clipped with Hem-O-loks without knotting. A homemade glove specimen bag was introduced, and all 

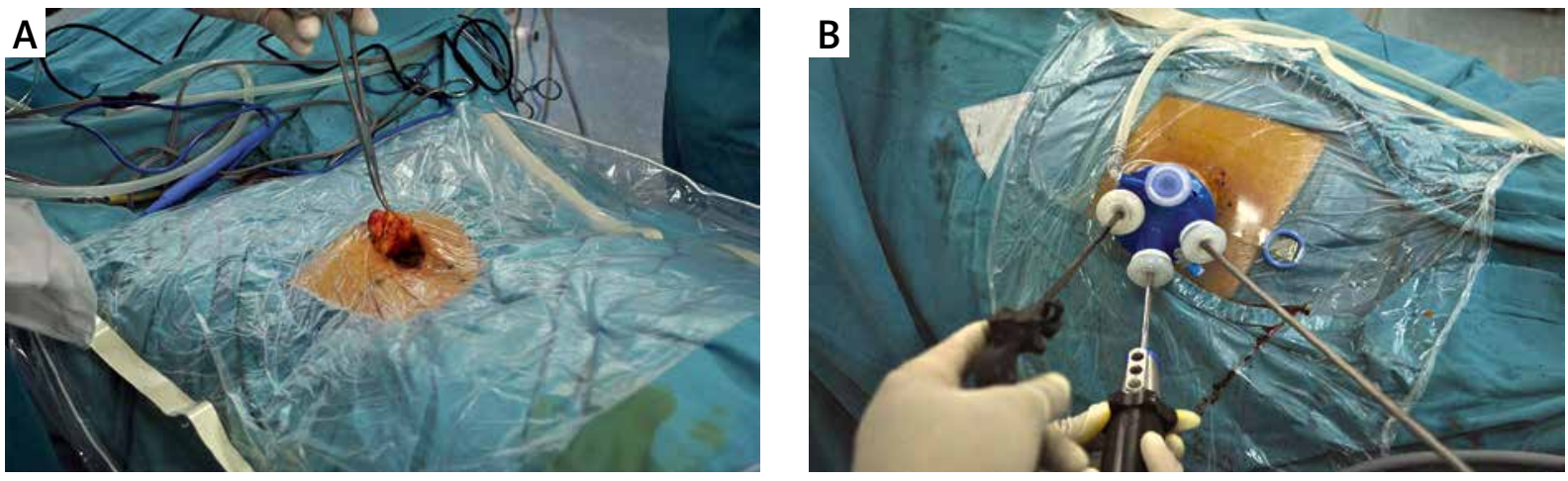

C

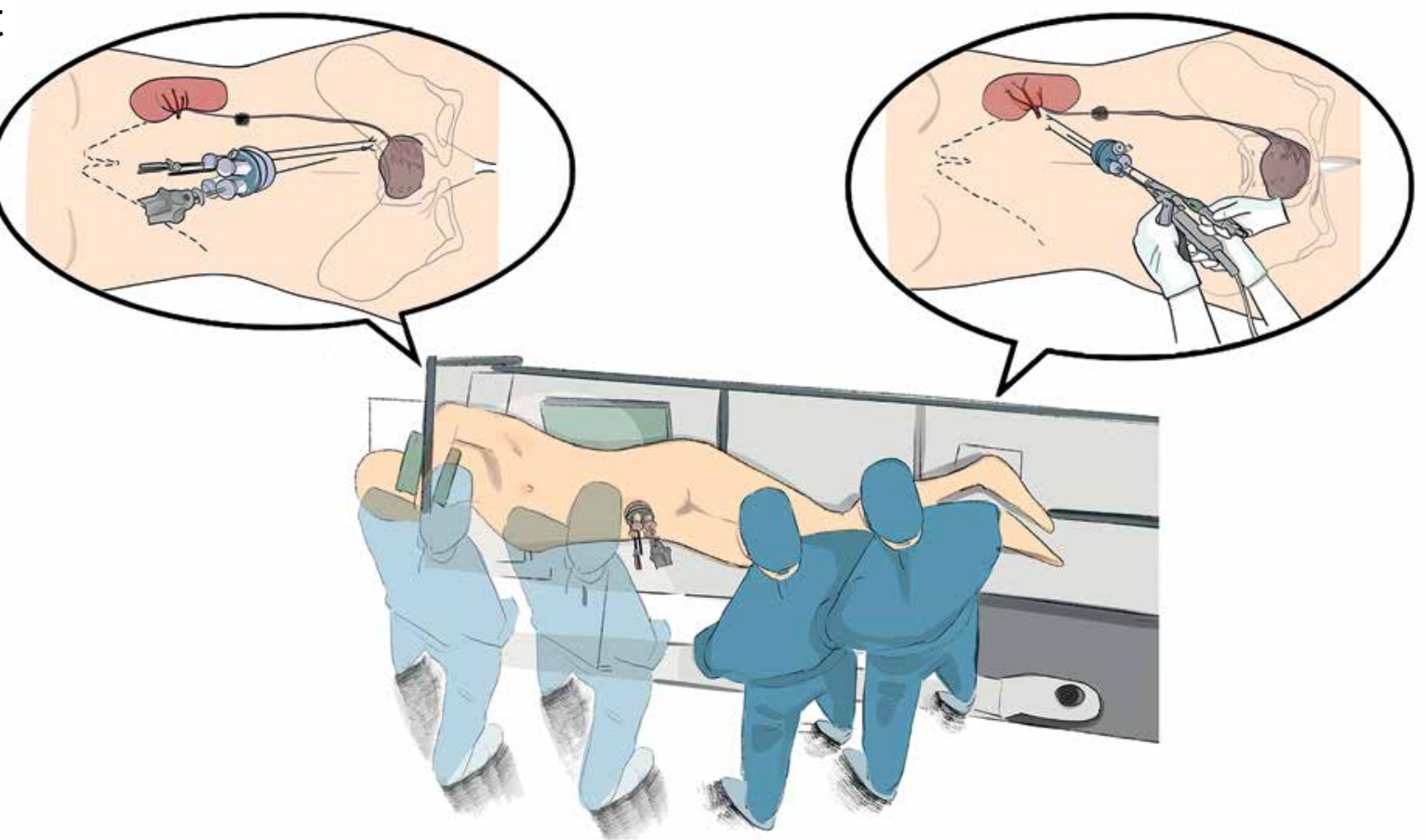

Figure 1. Operation preparation and patient position. A - Circumferential umbilical incision was made. B - The instruments were manipulated through the QuadPort multichannel port. C - Patient is placed with flank elevated by $30-45^{\circ}$. During the operation, surgeons can dissect the kidney and ureter directly continuously

specimens were retrieved en bloc in the bag via the umbilical incision. The drainage tubes were placed in the perirenal and pelvic cavity (Figures 2 C, D). Finally, a catheter was introduced into the bladder, the pneumoperitoneum was evacuated, and the port was removed. The skin incision was closed with absorbable sutures.

\section{Follow-up regime}

After the operation, patients were reviewed with a complete blood count, serum electrolyte, and cre- atinine tests and $\mathrm{CT}$ imaging for three months in the first year. In the following time of the review, all patients were contacted by phone to update their status. Cystoscopy was performed when patients were symptomatic or when investigations were abnormal.

\section{Statistical analysis}

Patient demographics, including age, gender, history of abdominal or pelvic surgery, American Society of Anesthesiologists score (ASA), and body mass index, were analyzed. 

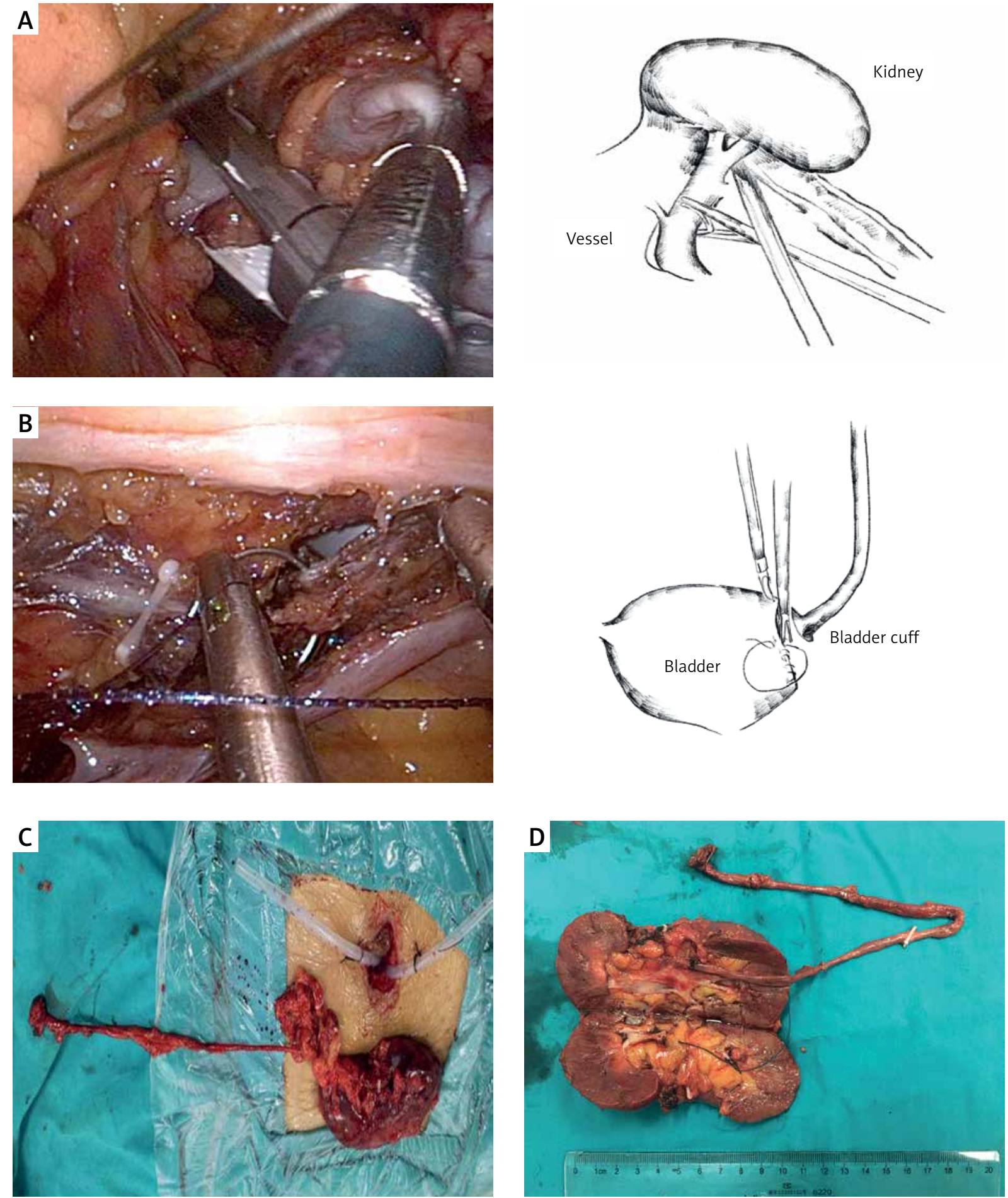

Figure 2. Key points in the LESS-NU procedures. A - The photo and diagram show the procedure of cutting off the renal vessel. B - The opened bladder wall was closed with running stitches by V-loc suture. $\mathbf{C}-$ The specimen is taken out through the patient's umbilicus incision. Two drainage tubes were placed in the perirenal and pelvic cavity through the wound. D - The specimen was taken with integrality 
Operative variables, including operating time, change in serum creatinine concentration, change in hemoglobin levels, conversion to open surgery, skin incision length, postoperative hospital stay, postoperative retention time of catheterization, visual analogue pain scale (VPS), and oral analgesic dose were analyzed. Clavien classification of surgical complications was used to grade postoperative complications.

Oncological outcomes, including tumor size, tumor location, pathological stage, tumor grade, margin status, and tumor recurrence, were analyzed. The 2002 TNM staging system was used to classify the tumor, and tumor grades were classified according to the 2004 WHO classification. Data were presented as mean \pm standard error of the mean. All data were compared using the Mann-Whitney $U$ test, $T$ test for continuous variables, and $\chi^{2}$ test for categorical variables. Statistical analysis was conducted using SPSS Statistics software version 24.0.

\section{Results}

According to Table I, a significant difference was found in patients' gender $(p=0.037)$. No significant differences in demographics were noted between the two groups. None of the patients had any previous abdominal or pelvic surgery. In comparison with the RL-NU group, a LESS-NU group patient required more trocars during the operation because

Table I. Patient demographics and operative variables of LESS-NU and RL-NU

\begin{tabular}{|c|c|c|c|}
\hline Parameter & LESS-NU $(n=25)$ & RL-NU $(n=17)$ & $P$-value* \\
\hline \multicolumn{4}{|l|}{ Gender, $n(\%)$ : } \\
\hline Female & $8(32)$ & $11(64.71)$ & 0.037 \\
\hline Male & $17(68)$ & $6(35.29)$ & \\
\hline Age, mean (SD) [years] & $67(9.24)$ & $69.12(9.28)$ & 0.626 \\
\hline BMI, mean (SD) [kg/m²] & $22.26(5.38)$ & $25.28(4.85)$ & 0.063 \\
\hline \multicolumn{4}{|l|}{ ASA score, $n(\%)$ : } \\
\hline 1 & $11(44)$ & $6(35.29)$ & 0.612 \\
\hline 2 & $12(48)$ & $8(47.06)$ & \\
\hline 3 & $2(8)$ & $3(17.65)$ & \\
\hline Previous abdominal or pelvic surgery, $n$ & 0 & 0 & \\
\hline Operating time, mean (SD) [min] & $204.4(36.26)$ & $236.18(24.41)$ & 0.005 \\
\hline Change in serum creatinine concentration, mean [mg/dl] & 8.87 & 9.57 & 0.838 \\
\hline Change in serum hemoglobin level, mean [g/dl] & 11.84 & 19.64 & 0.140 \\
\hline Postoperative retention time of catheterization, mean [days] & 3.88 & 3.76 & 0.327 \\
\hline Surgery conversion, $n$ (\%) & $1(4)$ & 0 & \\
\hline Skin incision length, mean (SD) [cm] & $2.88(0.43)$ & $5.94(2.63)$ & $<0.001$ \\
\hline Visual analogue pain scale, mean (SD) & $2(0.57)$ & $3.76(1.06)$ & $<0.001$ \\
\hline Oral analgesic dose, mean (SD) [days] & $1.12(0.65)$ & $2.75(0.82)$ & $<0.001$ \\
\hline Intraoperative complications, $n$ (\%) & 0 & 0 & \\
\hline Postoperative complications, $n$ : & & & 0.849 \\
\hline Grade 1 & 5 & 3 & \\
\hline Grade 2 or $>2$ & 0 & 0 & \\
\hline
\end{tabular}

*Mann-Whitney $U$ test for continuous variables and the $\chi^{2}$ test for categorical variables. 
of obesity. However, the mean operation time of the LESS-NU group was significantly shorter than that of the RL-NU group $(p=0.006)$. The mean skin incision length was also shorter in the LESS-NU group $(p<0.001)$. Additionally, the mean VPS of LESS-NU was significantly lower than that of the RL-NU group $(p<0.001)$.The LESS-NU group patients required fewer oral analgesics than the RL-NU group patients. Overall, no significant difference was found in the change in serum creatinine concentration and hemoglobin levels between the two groups ( $p=0.838$ and $p=0.14$, respectively). No significant differences were also found in postoperative hospital stay ( $p=$ $0.797)$ and in postoperative retention time of catheterization $(p=0.805)$ and ASA score $(p=0.612)$. Postoperative complication syndrome occurred in 11 patients (LESS-NU, $n=7$; RL-NU, $n=4$ ). Four patients who belonged to the LESS-NU group had fever after surgery, and one patient in the LESS-NU group suffered abdominal pain. In the RL-NU group, pneumonia was handled with a conservative anti-infection treatment, and two other patients had postoperative fever. All these cases were resolved with conservative management.

All the 42 patients had confirmed UTUC based on the pathological results. The major tumor was in the T3 stage ( $n=20,47.62 \%)$ according to Table II. Low tumor grade was observed in $6(14.29 \%)$ patients. One pN2 nodal status and one positive margin status were both noted in the RL-NU group. No significant differences were obtained in the pathological characteristics between the two groups.

The mean follow-up was 31.64 months, and no significant difference was observed between the two groups (Table III). Among the 33 continuous follow-up patients, two patients died during our

Table II. Oncological characteristics

\begin{tabular}{|c|c|c|c|}
\hline Parameter & LESS-NU $(n=25)$ & RL-NU $(n=17)$ & $P$-value \\
\hline Preoperative clinical T stage, $n(\%)$ : & & & 0.206 \\
\hline cTa & 0 & $1(5.88)$ & \\
\hline cT1 & $5(20)$ & $6(35.29)$ & \\
\hline cT2 & $8(32)$ & $2(11.76)$ & \\
\hline сT3 & $12(48)$ & $8(47.06)$ & \\
\hline Tumor side, $n$ (\%): & & & 0.206 \\
\hline Left & $7(28)$ & $8(47.06)$ & \\
\hline Right & $18(72)$ & $9(52.94)$ & \\
\hline Tumor site, $n(\%)$ : & & & 0.6 \\
\hline Upper or middle ureteric & $8(32)$ & $3(17.65)$ & \\
\hline Lower ureteric & $4(16)$ & $4(23.53)$ & \\
\hline Pelvocalyceal & $10(40)$ & $9(52.94)$ & \\
\hline Multifocal & $3(12)$ & $1(5.88 \%)$ & \\
\hline Tumor grade, $n(\%)$ : & & & 0.158 \\
\hline Low & $2(8)$ & $4(23.53)$ & \\
\hline High & $23(92)$ & $13(76.47)$ & \\
\hline Nodal status, $n(\%)$ : & & & 0.158 \\
\hline $\mathrm{pNx}$ & $15(60)$ & $7(5.88)$ & \\
\hline pNO & $10(40)$ & $9(52.94)$ & \\
\hline pN1-3 & 0 & $1(5.88 \%)$ & \\
\hline
\end{tabular}

*Mann-Whitney $U$ test and $T$ test for continuous variables and the $\chi^{2}$ test for categorical variables. 
Table III. Follow-up outcomes of patients in two groups

\begin{tabular}{|lccc|}
\hline Parameter & LESS-NU $(n=25)$ & RL-NU $(n=17)$ & $P$-value \\
\hline Follow-up, mean (SD) [months] & $31.57(8.195)$ & $32.75(10.506)$ & 0.722 \\
\hline Follow-up persons, $n$ & 21 & 12 & 0.876 \\
\hline Chemotherapy, $n(\%)$ & $18(90)$ & $11(91.67)$ & 3 \\
\hline Tumor recurrence rate, $n:$ & 5 & $1(8.33)$ & \\
\hline Bladder recurrence, $n(\%)$ & $2(9.52)$ & $1(8.33)$ & $1(8.33)$ \\
\hline Pelvic metastasis, $n(\%)$ & $2(9.52)$ & $1(4.76)$ &
\end{tabular}

follow-up period. Both patients were in the RL-NU group, including the pN2 patient. The pN2 patient died of pelvic metastasis 15 months after the operation. Another patient also died of a cancer-related cause after the 1-year follow-up. Overall, recurrences occurred in 8 patients, and no difference was observed between the two groups.

\section{Discussion}

The conventional open nephroureterectomy (ONU) procedure, which is the earliest method of surgery, requires a lumbar incision at the affected side [14]. The kidney and middle and upper ureter were resected through the incision. Then, another large McBurney incision at the affected side was made to resect the distal ureter and bladder cuff. However, this method has been replaced by several minimally invasive procedures because of its wound disadvantage. Since Clayman et al. reported the first successful laparoscopic nephroureterectomy in 1991, minimally invasive approaches have been performed in urological surgery [15]. In recent years, the retroperitoneal approach has become more beneficial than the other approaches [16] and has become a major conventional surgical procedure to treat UTUC. Surgeons prefer to maximize the clinical effect with minimal invasiveness. LESS-NU surgery was introduced as a novel and safe procedure. Our department began to perform LESS in 2009 [10, 11]. Sufficient clinical experience has been accumulated in recent years.

In this study, we designed a modified LESS-NU procedure and made a comparison with the RL-NU procedure in our department. Through this method, we tried to compare the advantages and disadvantages between modified LESS approach and retroperitoneal approach. To our knowledge, this report is the first analysis of these two procedures on the management of UTUC.

On the management of the surgical procedure, many institutions have reported their own modifications and innovation [17, 18]. By summarizing our previous experience, we agreed that separating the renal pedicle was the key step. First, the paracolic sulci needed to be separated as much as possible. The colon would slide downward under the action of gravity. At this moment, the renal hilum would be exposed maximally. When isolating the renal vein, the vein should be bared in parallel with the renal vein path. And, blunt separate and sharp separate could be combined to separate the surrounding tissue. When producing the right side, the fourth channel on the single port can be used to protect the low edge of the liver, vena cava, and duodenum. In our current series, we were able to separate the kidney and dissect the ureter down the bladder cuff region. Additionally, the V-loc suture can be chosen to close the bladder cuff without knotting. It is a direct method to handle the problem of knotting. In the largest multi-institutional series, Park et al. showed that their mean operation time was $221 \mathrm{~min}$ [19]. In their series, $20.8 \%$ of the cases were performed without bladder cuff excision. In the latest LESS-NU study, Tsivian et al. had a mean operation time of $217 \mathrm{~min}$ [20]. In the present series of 25 LESS-NU cases, the mean operation time was 204.4 min, which was shorter than those of the above studies. By contrast, all patients in the RL-NU group needed to be repositioned during the operation. It potentially wasted 20-30 min of operating time for altering the patient's position, disinfecting, and toweling.

Based on the previous description [12, 13], our RL-NU procedure managed 3-4 ports on the waist. 
Incisions may be extended to $5-7 \mathrm{~cm}$ for specimen extraction. The length of incision was mainly related to the patient's postoperative pain. In our LESS-NU group, we chose the umbilicus as this single port, because the tissue surrounding the umbilicus was thin. All procedures, such as kidney and ureter dissection, distal ureter and bladder cuff excision, and bladder closure, were performed via this port. It meant that injuries to the abdominal wall and the postoperative pain could be reduced. Identifying the anatomical sign and obtaining a clear operative view were easily done by settling a pneumoperitoneum. Additionally, the use of a flexible single port could ease the manipulation through the transumbilical approach. Owing to the small incision and little tension around the umbilical port, the 3-0 absorbable sutures could be used to make intermittent suture or running suture. Sutures that would reduce the length of stay need not be removed. Due to the umbilicus being a natural fold part of human body, the wound surrounding the umbilicus was inconspicuous. A "scarless" procedure was partially accomplished by the transumbilical LESS procedure. In the present series, the mean incision length of LESS-NU patients was $2.88 \mathrm{~cm}$. By contrast, a $8.94 \mathrm{~cm}$ mean large lower abdominal oblique incision was required in the RL$\mathrm{NU}$ group. It was a huge improvement in the management of the operation wound.

At the same time, the size of the wound could directly affect the patient's postoperative pain. Bansal et al. also agreed with the potential cosmetic benefits of this minimally invasive surgery [21]. According to our analysis, the length and number of skin incisions had a positive correlation with the VPS and oral analgesic dose. This result was in accordance with some previous reports. Fan et al. found that owing to the obvious reduction of the surgical wound, the postoperative pain would be significantly reduced compared with the traditional laparoscopic surgery [5]. In our study, patients in the LESS-NU group required an oral analgesic for 1 or 2 days. However, patients who underwent RL$\mathrm{NU}$ required more oral analgesic due to the large wound. The reduction of the postoperative pain is helpful to evaluate the postoperative patient's recovery and predict the postoperative complications. During our follow-up, some patients who underwent RL-NU reported ongoing pain on their wounds for almost 1 month after the operation despite the recovery of the wound. Faint pain was also reported on rainy days. In the LESS-NU group, a few patients felt pain after leaving the hospital. Overall, the patients were more satisfied with the LESS-NU for its advantages of postoperative recovery and less pain. Olweny et al. firstly evaluated the patients' perceptions of the scarring relative to other surgical outcomes [22]. Current patients paid more attention to hospitalization experience, scar length, postoperative cosmetic effect, and postoperative quality of life. The LESS-NU procedure met these patients' needs.

Interestingly, we found a significant difference in gender. However, the population of women choosing LESS-NU per year increased. With the development and maturity of our LESS-NU technique, more women favored the LESS-NU procedure. UTUC is more popular in female patients (55.4\%) than male patients (44.6\%) in China [23]. We agreed that LESSNU would be a perfect choice for female patients because of its better cosmesis and less pain.

In our follow-up period, 33 patients continued reviewing our series until we finished this report. Connections with the nine other patients were lost because of their missing phone numbers or moving to other places. The mean follow-up time was 31.64 months. During this time, 2 patients died of cancer-related causes in the RL-NU group. A patient was pathologically confirmed with PT3 N2 cMo status. When this patient left the hospital for 6 months, he had a confirmed pelvic metastasis. Another patient was diagnosed with bone metastasis. Additionally, 10 metastasis cases were checked. These metastasis patients were still alive and receiving chemotherapy actively. For the short-term review, LESS-NU attained the same achievement as the RL-NU procedure.

The limitations of our study should be noted. The study was limited by its relatively small retrospective sample size, and patient selection between LESS-NU and RL-NU was not randomized. The benefits of LESS-NU should be demonstrated through a large number of trials, and oncology outcomes should be analyzed by conducting a long-term follow-up review. Despite the limitations, this study is still the first to make the comparison between the modified LESS-NU and RL-NU. Meanwhile, we introduce a modified, minimally invasive technique. We believe that our experience will attract more surgeons to perform our modified LESS-NU and help patients benefit from this technique. 


\section{Conclusions}

This study provided the first comparison between the modified LESS-NU and RL-NU. We introduced a modified and minimally invasive technique. We believe that our experience will attract more surgeons to perform modified LESS-NU, which will benefit patients.

\section{Acknowledgments}

Yang Shen, Hesong Ye and Qingyi Zhu were cofirst authors.

The authors gratefully acknowledge the support of the urology department of Jiangsu Provincial Hospital of Chinese Medicine.

\section{Conflict of interest}

The authors declare no conflict of interest.

\section{References}

1. Ljungberg B, Cowan NC, Hanbury DC, et al. EAU guidelines on renal cell carcinoma: the 2010 update. Eur Urol 2010; 58 : 398-406.

2. Babjuk M, Sylvester R, Oosterlinck W. Guidelines on upper urinary tract urothelial cell carcinomas. Eur Urol 2011; 59: 584-94.

3. Kavoussi LR, Kerbl K, Capelouto CC, et al. Laparoscopic nephrectomy for renal neoplasms. Urology 1993; 42: 603-9.

4. Rassweiler JJ, Schulze M, Marrero R, et al. Laparoscopic nephroureterectomy for upper urinary tract transitional cell carcinoma: is it better than open surgery? Eur Urol 2004; 46: 690-7.

5. Fan X, Lin T, Xu K, et al. Laparoendoscopic single-site nephrec tomy compared with conventional laparoscopic nephrectomy: a systematic review and meta-analysis of comparative studies. Eur Urol 2012; 62: 601-12.

6. Aull MJ, Afaneh C, Charlton M, et al. A randomized, prospective, parallel group study of laparoscopic versus laparoendoscopic single site donor nephrectomy for kidney donation. Am J Transpl 2014; 14: 1630-7.

7. White WM, Haber GP, Goel RK, et al. Single-port urological surgery: single-center experience with the first 100 cases. Urology 2009; 74: 801-4.

8. White WM, Goel RK, Kaouk JH. Single-port laparoscopic retroperitoneal surgery: initial operative experience and comparative outcomes. Urology 2009; 73: 1279-82.

9. Zhu C, Su J, Yuan L, et al. Transurethral assistant transumbilical laparoendoscopic single-site radical prostatectomy. Asian J Androl 2017; 19: 473-6.

10. Su J, Zhu Q, Yuan L, et al. Combined laparoendoscopic single-site ureterolithotomy and flexible cystoscopy in the treatment of concurrent large upper ureteral and renal stones. Scand J Urol 2017; 51: 314-8.

11. Su J, Zhu Q, Yuan L, et al. Transumbilical laparoendoscopic single-site radical prostatectomy and cystectomy with the aid of a transurethral port: a feasibility study. BJU Int 2018; 121: 111-8.
12. Goel A, Hemal AK, Gupta NP. Retroperitoneal laparoscopic radical nephrectomy and nephroureterectomy and comparison with open surgery. World J Urol 2002; 20: 219-23.

13. Matsui Y, Ohara H, Ichioka K, et al. Retroperitoneoscopy-assisted total nephroureterectomy for upper urinary tract transitional cell carcinoma. Urology 2002; 60: 1010-5.

14. Bonjer HJ, Hazebroek EJ, Kazemier G, et al. Open versus closed establishment of pneumoperitoneum in laparoscopic surgery. Br J Surg 1997; 84: 599-602.

15. Waldert M, Remzi M, Klingler HC, et al. The oncological results of laparoscopic nephroureterectomy for upper urinary tract transitional cell cancer are equal to those of open nephroureterectomy. BJU Int 2009; 103: 66-70.

16. Choi KH, Ham WS, Rha KH, et al. Laparoendoscopic single-site surgeries: a single-center experience of 171 consecutive cases. Korean J Urol 2011, 52: 31-8.

17. Seo IY, Hong HM, Kang IS, et al. Early experience of laparoendoscopic single-site nephroureterectomy for upper urinary tract tumors. Korean J Urol 2010; 51: 472-6.

18. Lee JY, Kim SJ, Moon HS, et al. Initial experience of laparoendoscopic single-site nephroureterectomy with bladder cuff excision for upper urinary tract urothelial carcinoma performed by a single surgeon. J Endourol 2011; 25: 1763-8.

19. Park SY, Rha KH, Autorino R, et al. Laparoendoscopic single-site nephroureterectomy for upper urinary tract urothelial carcinoma: outcomes of an international multi-institutional study of 101 patients. BJU Int 2013; 112: 610-5.

20. Tsivian A, Tsivian M, Stanevsky Y, et al. Laparoendoscopic single-site plus one port nephroureterectomy: single center initial experience. J Endourol 2014; 28: 675-8.

21. Bansal D, Cost NG, Bean CM, et al. Comparison of pediatric robotic-assisted laparoscopic nephroureterectomy and laparoendoscopic single-site nephroureterectomy. Urology 2014; 83: 438-42.

22. Olweny EO, Mir SA, Best SL, et al. Importance of cosmesis to patients undergoing renal surgery: a comparison of laparoendoscopic single-site (LESS), laparoscopic and open surgery. BJU Int 2012; 110: 268-72.

23. Xiong G, Chen X, Li X, et al. Prevalence and factors associated with baseline chronic kidney disease in China: a 10-year study of 785 upper urinary tract urothelial carcinoma patients. J Form Med Assoc 2014; 2014: 521-6.

Received: 14.05.2019, accepted: 3.07.2019. 